\title{
Onset of high- $n$ ballooning modes during tokamak sawtooth crashes
}

\author{
Y. Nishimura, ${ }^{\text {a) }}$ J. D. Callen, and C. C. Hegna \\ Department of Engineering Physics, University of Wisconsin-Madison, Madison, Wisconsin 53706-1687
}

(Received 27 July 1999; accepted 3 September 1999)

\begin{abstract}
A new phenomenon has been found during the nonlinear stage of the tokamak sawtooth crash in relatively high $\beta$ plasmas. The $m / n=1 / 1$ magnetic island evolution gives rise to convection of the pressure inside the $q=1$ radius and builds up steep pressure gradient across the island separatrix, and thereby trigger ballooning instabilities below the threshold at the equilibrium. Effects of the ballooning modes on the magnetic reconnection process during the sawtooth crash are discussed. (C) 1999 American Institute of Physics. [S1070-664X(99)02812-8]
\end{abstract}

\section{INTRODUCTION}

Sawtooth oscillations ${ }^{1}$ are generally observed in tokamak plasmas when the safety factor $q$ at the magnetic axis is below unity. The temporally discontinuous soft $x$-ray signals suggest sudden electron temperature drops or crashes in the core of the plasma column. After the crash, the temperature profile inside the $q=1$ radius is flattened. [Some exceptions of sawtooth free discharges are reported in Toroidal Experiment for Technically Oriented Research (TEXTOR) ${ }^{2}$ and Tokamak Fusion Test Reactor (TFTR) supershots. ${ }^{3}$ ]

There is a large gap between the theoretical understanding and the experimental results; the mechanism responsible for the nonlinear stage of the crash phase is far from being understood. The following two principal problems remain unsolved for sawtooth studies: (1) The central $q$-value remains below unity during the crash in most experiments ${ }^{2}$ whereas zero-pressure Kadomtsev model predicts ${ }^{4}$ a full magnetic field reconnection and that the central $q$ rises to above unity in the core of the plasma. (2) The time scale of the final crash phase is much shorter than the one predicted by resistive reconnection ${ }^{4}$ or the Sweet-Parker layer model. ${ }^{5}$ In today's high-temperature tokamak plasmas, the time scale is on the order of tens of microseconds. ${ }^{6}$

Recent experimental results, for example, those in Refs. 7 and 8, indicate dynamical temperature evolution during the crash phase of a sawtooth oscillation. Furthermore, the experimental measurements in TFTR $^{6}$ have suggested the mode localization of the pressure driven modes (ballooning modes) on the bad curvature side of the torus.

On top of the ballooning mode localization, a series of TFTR experiments ${ }^{9,10}$ as well as TEXTOR experiments ${ }^{11}$ have inferred that the magnetic field line stochasticity in the vicinity of the $q=1$ radius plays an important role in thermal transport and the resultant rapid changes in the magnetic field structure. In the experiment, ${ }^{10}$ it is suggested that the pressure contours and the magnetic flux surface does not coincide during the nonlinearly developed stages of the crash.

In this work, numerical simulation results on the excita-

\footnotetext{
${ }^{\text {a) }}$ Present address: Department of Physics, University of Colorado, Boulder, Colorado 80309-0390. Electronic mail: nishimya@colorado.edu
}

tion of the secondary high- $n$ ballooning modes will be presented. Quantitative analysis of enhanced magnetic field line stochasticity is discussed in a separate paper. ${ }^{12}$

This paper is organized as follows. In Sec. II the basic model for magnetohydrodynamic (MHD) simulation is discussed. In Sec. III, we present results of MHD simulations on the onset of high- $n$ ballooning modes, showing evolution of both the pressure and magnetic field structures. Toroidal asymmetry of the $m=1$ magnetic island structure is discussed in Sec. IV. We summarize this work in Sec. V.

\section{BASIC MODEL FOR COMPUTATION}

In this section, the basic properties of a magnetic field and a reduced MHD formulation in a tokamak are reviewed. A nonorthogonal, straight-field-line coordinate system is employed in the present calculations, in which $\rho$ is the flux surface label, $\theta$ is the poloidal-like angle, and $\zeta$ is the toroidal angle. The magnetic field in a tokamak can be written as

$$
\begin{aligned}
& \mathbf{B}=\mathbf{B}_{\mathbf{e q}}+\widetilde{\mathbf{B}}, \\
& \mathbf{B}_{\mathbf{e q}}=-F \nabla \zeta+\nabla \zeta \times \nabla \psi_{\text {eq }}, \\
& \widetilde{\mathbf{B}} \equiv \nabla \times \widetilde{\mathbf{A}}=\nabla \times(-\widetilde{\psi} \nabla \zeta)=\nabla \zeta \times \nabla \widetilde{\psi},
\end{aligned}
$$

where $\psi_{\mathrm{eq}}(\rho)$ and $F(\rho)$ stand for the equilibrium poloidal magnetic flux and equilibrium poloidal current. Here, $\widetilde{\psi}(\rho, \theta, \zeta)=\Sigma_{m / n} \widetilde{\psi}_{m / n}(\rho) \cos (m \theta+n \zeta)$ is the poloidal flux function of the perturbed field, where $m$ and $n$ are the poloidal and the toroidal mode numbers, respectively. We denote the total poloidal magnetic flux as $\psi(\rho, \theta, \zeta) \equiv \psi_{\text {eq }}(\rho)$ $+\widetilde{\psi}(\rho, \theta, \zeta)$.

Numerical simulation has been conducted employing a reduced MHD formulation ${ }^{13}$ in a toroidal geometry. Toroidal geometry enters via metric elements obtained from an equilibrium solver RSTEQ. ${ }^{14}$ The initial value simulation is conducted by employing the FAR code ${ }^{15}$ which originally contained a full set of MHD equations. In our study the reduced MHD equations ${ }^{13}$ are solved for the magnetic flux $\psi$, the toroidal component of the vorticity $U^{\zeta}$, and the pressure $p$. The relevant equations are: the toroidal component of Ohm's law, 


$$
\frac{\partial \psi}{\partial t}=-R^{2}\left[(\mathbf{v} \times \mathbf{B}) \cdot \nabla \zeta-\eta J^{\zeta}\right],
$$

the vorticity equation,

$$
\begin{aligned}
\frac{d U^{\zeta}}{d t}= & S^{2} R^{2}\left[\mathbf{B} \cdot \nabla\left(\frac{J_{\zeta}}{F}\right)+\frac{\beta}{2 \epsilon^{2}}\left(\nabla \frac{1}{B^{\zeta}} \times \nabla p\right) \cdot \nabla \zeta\right] \\
& +\nu_{\perp} \nabla^{2} U^{\zeta},
\end{aligned}
$$

and the pressure evolution equation,

$$
\frac{d p}{d t}=\kappa_{\perp} \nabla_{\perp}^{2} p .
$$

In these equations, $R$ stands for the major radius, $\mathbf{v} \equiv R^{2} \nabla \zeta$ $\times \nabla \phi$ is the fluid velocity ( $\phi$ is the stream function), $\mathbf{U}$ $\equiv R^{2} \nabla \times\left(R^{2} \mathbf{v} / F\right)$ is the vorticity, and $\mathbf{J} \equiv \nabla \times \mathbf{B}$ is the current density. Superscripts (subscripts) denote the contravariant (covariant) components. Here $\eta$ is the resistivity and the transport coefficients are given by $\nu_{\perp}$, and $\kappa_{\perp}$, which are the perpendicular viscosity and the perpendicular heat conductivity, respectively. In this work heat conduction parallel to the magnetic field is ignored due to numerical difficulties. The maximum toroidal beta value at the magnetic axis is denoted by $\beta$, while the inverse aspect ratio is $\epsilon$. The reduced MHD formulation is derived by neglecting $\epsilon^{2}$ and higherorder terms and is correct to the small inverse aspect ratio limit where $\epsilon \ll 1$.

Time is normalized by the resistive time $\tau_{r} \equiv a^{2} / \eta$ and the length is normalized by the minor radius $a$. The Lundquist number is given by $S \equiv \tau_{r} / \tau_{a}$ where $\tau_{a}$ is the poloidal Alfven transit time. Incompressibility $\left(\nabla \cdot R^{-2} \mathbf{v}=0\right)$ is implicit in Eq. (6). Toroidal curvature effects are included in the second term of Eq. (5), which is the minimum requirement to describe the ballooning-type pressure driven modes. The reduced MHD does not precisely include the effect of the $n=1$ toroidal internal kink mode. The analysis by Bussac et al. ${ }^{16}$ reveals that the mode enters through order $\epsilon^{4}$ in the variational principle. ${ }^{16}$ Bussac et al. ${ }^{16}$ have shown that the internal kink mode is only unstable when $\beta_{p, \text { Bussac }}>0.3$ ( $\beta_{p \text {, Bussac }}$ is the poloidal beta value which is volume averaged within the $q=1$ radius, and differs from the conventional poloidal beta $\beta_{p}$ ). In our simulation, possibly due to the absence of higher-order term effects, the $m / n=1 / 1$ ideal/ resistive kink modes are always linearly unstable regardless of what $\beta$ value one employs.

To time advance the reduced MHD equations, the unknown quantities $x(\rho, \theta, \zeta)$ in Eqs. (4), (5), and (6) are separated into equilibrium and perturbation parts: $x(\rho, \theta, \zeta)$ $=x_{\mathrm{eq}}(\rho, \theta)+\tilde{x}(\rho, \theta, \zeta)$. The purely equilibrium terms are discarded in the FAR code,,${ }^{15}$ leaving only terms that are linear or quadratic in the perturbed quantities. Linear calculations time advance only the $x_{\mathrm{eq}} \times \tilde{x}$ terms implicitly, while nonlinear calculations integrate quadratic terms $\tilde{x} \times \tilde{x}$ explicitly. The perturbed quantities $\tilde{x}(\rho, \theta, \zeta)$ are finite differenced in $\rho$ and expanded in Fourier series for the poloidal angle $\theta$ and the toroidal angle $\zeta$. At $t=0$ we perturb a single resonant mode $\phi_{1 / 1}$ with a finite amplitude. To see the evolution of

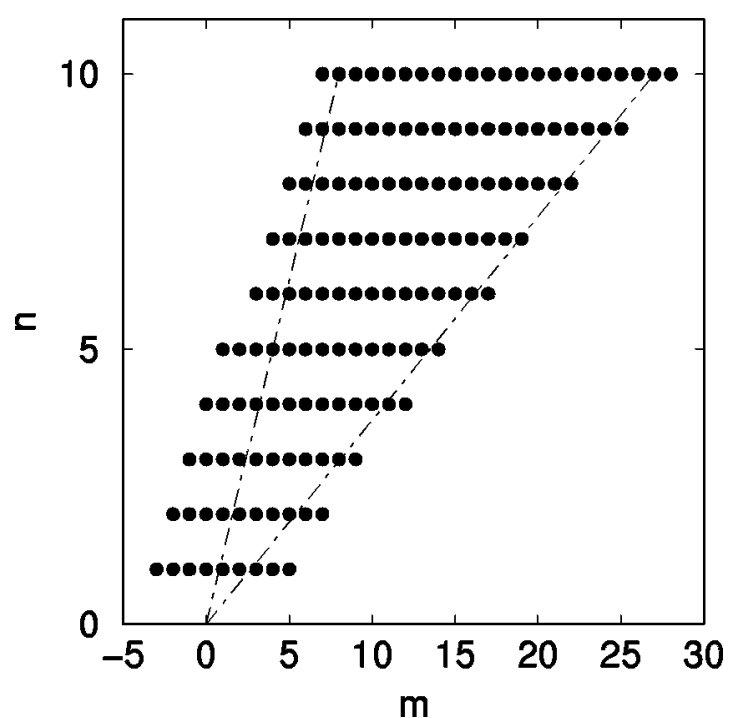

FIG. 1. Mode spectrum employed for the simulation. The abscissa and ordinate corresponds to poloidal and toroidal mode numbers, respectively. A total of 151 modes are used in the simulations.

magnetic structures, magnetic field line trajectories have been obtained by integrating the magnetic field line equation

$$
\begin{aligned}
& \frac{d \rho}{d \zeta}=\frac{B^{\rho}}{B^{\zeta}}=-\frac{1}{\rho F} \frac{\partial \psi}{\partial \theta}, \\
& \frac{d \theta}{d \zeta}=\frac{B^{\theta}}{B^{\zeta}}=\frac{1}{\rho F} \frac{\partial \psi}{\partial \rho},
\end{aligned}
$$

using a fourth-order Runge-Kutta-Gill method. ${ }^{17}$

\section{SIMULATION RESULTS}

Parameters used in the calculations were as follows. The $q$-profile is taken as a peaked profile. ${ }^{18}$ The central- $q$ is taken as $q_{0}=0.81$ to avoid the resonance of $m / n=4 / 5$ mode near the magnetic axis. The equilibrium pressure profile has the form of $p=\psi_{\mathrm{eq}}^{2}$. Major radius $R=5 \mathrm{~m}$, minor radius $a$ $=1.25 \mathrm{~m}$ (the inverse aspect ratio $\epsilon=1 / 4$ ), and Lundquist number $S=10^{5}$ are taken. A total of 500 equally spaced mesh points was used in the radial direction. The mode spectrum was selected as shown in Fig. 1, so that it fills the $0.81 \leqslant q \sim m / n \leqslant 2.7$ region. A total of 151 modes was included in the simulations. The transport coefficients are taken as small as possible: $\nu_{\perp}=0.1$, and $\kappa_{\perp}=0.1$.

Figures 2(a) and 2(b) show pressure contours in the poloidal cross sections at $\zeta=\pi$ and $\zeta=0$ after a nonlinear evolution of $m=1$ magnetic island. All the figures in this paper are plotted in the Cartesian (configuration space) coordinates $(X, Z)$. The center line of the torus is located at the middle of the figure and the toroidal magnetic field falls off proportional to $1 / R$ toward the edges. In Fig. $2, \beta$ of $4 \%$ (poloidal beta of $\beta_{p}=0.74$ ) was used. The green and yellow crescentshaped part corresponds to the magnetic island. Localization of the mode on the bad curvature side can be seen starting from $t=1.205 \times 10^{-2}$; the $m / n=1 / 1$ magnetic island evolution gives rise to convection of the pressure inside the $q$ $=1$ radius and builds up a steep pressure gradient across the 
(a)

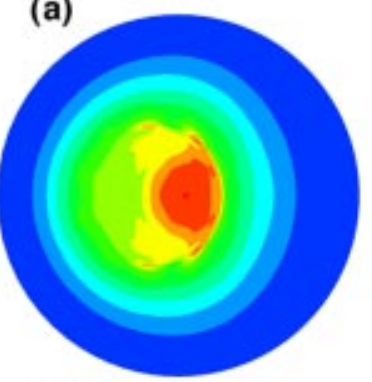

(c)

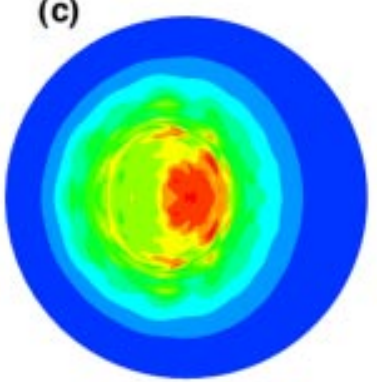

(e)

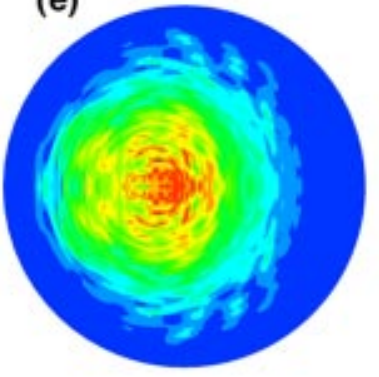

(b)

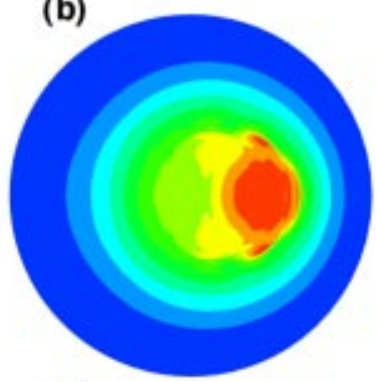

(d)

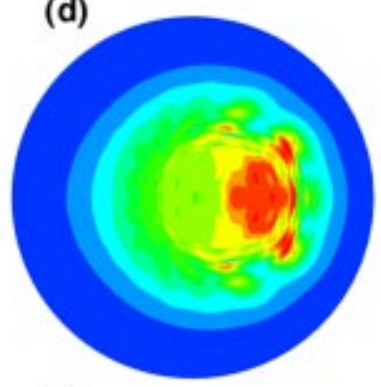

(f)

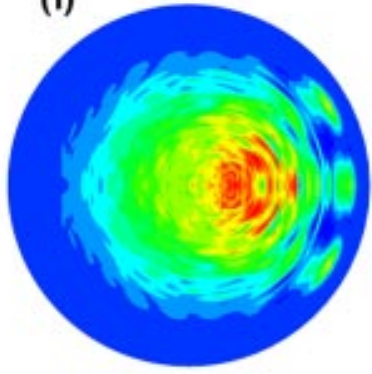

FIG. 2. Pressure contours for the $\beta=4 \%$ simulation. The axisymmetry center line is located at the middle of the page. (a), (b) at $t=1.205 \times 10^{-2}$, (c), (d) at $t=1.230 \times 10^{-2}$, and (e), (f) at $t=1.265 \times 10^{-2}$.

island separatrix or the Y-ribbon, ${ }^{19}$ and thereby triggers ballooning instabilities below the threshold at the equilibrium. At later times $\left(t=1.230 \times 10^{-2}\right.$ and $\left.t=1.265 \times 10^{-2}\right)$, typical ballooning type structures (so-called ballooning fingers) on the bad curvature side can be seen. Note that the corresponding high- $n$ mode activity is suppressed in Figs. 2(a), 2(c), and 2(e) where the hot core is facing the good curvature side. Figures 2(e) and 2(f) show the final temperature crash phase.

Figure 3 shows eigenprofiles of the streamfunction $\phi$ : the commensurate $1 / 1$ to $10 / 10$ mode spectrum, at $t=1.175$ $\times 10^{-2}$ when the high- $n$ mode amplitudes are small, and at $t=1.230 \times 10^{-2}$ when the high- $n$ modes are active. Figure 3 indicates rapid growth of the $8 / 8$ to $10 / 10$ modes (red, blue, and green curves).

Figure 4 shows eigenprofiles of $n=10$ modes but with different $m$ (poloidal mode) numbers. These incommensurate helicity modes grow simultaneously. This is a typical eigenmode structure of ballooning modes; different $m$ modes with same $n$ are correlated in the ballooning transformation. ${ }^{20}$ Note that each of the modes are localized near the corresponding mode rational surfaces exhibiting the nature of Weber functions ${ }^{21}$ - say $15 / 10$ at $q=1.5$ surface (red), 16/10 at $q=1.6$ (blue), $17 / 10$ at $q=1.7$ (green) ..., and so on.

(a)

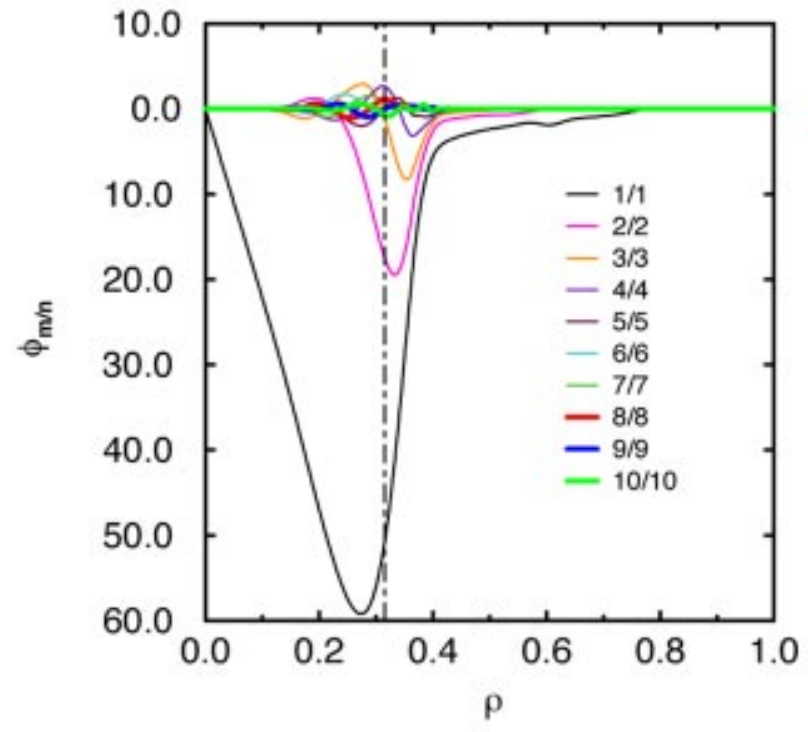

(b)

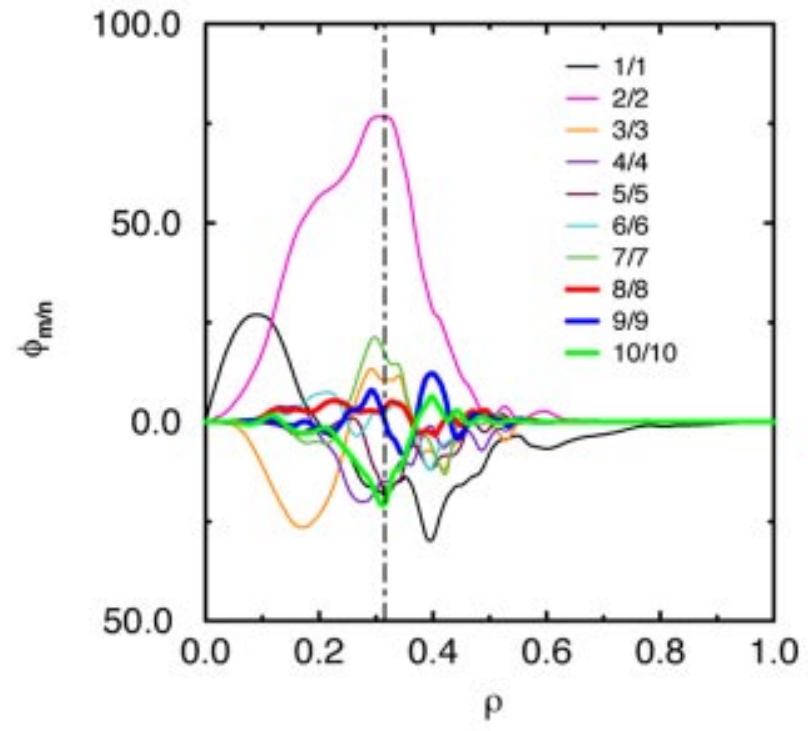

FIG. 3. Fourier spectrum of the stream functions $\phi$ (a) at $t=1.175 \times 10^{-2}$ and (b) at $t=1.230 \times 10^{-2}$. Commensurate modes, $m / n=1 / 1$ to $m / n$ $=10 / 10$.

An important observation is that these higher number modes are not active in the axisymmetric equilibrium but are triggered by the change in the pressure profile in the vicinity of the Y-ribbon. ${ }^{19}$ These high- $n$ modes are not generated from mode coupling but rather they result from changes in the $n=0$ equilibrium modes. The ideal $n=10$ ballooning modes are stable to $\beta \sim \epsilon$ which is allowed for the reduced MHD description. ${ }^{13}$ The resistive ballooning modes ${ }^{22}$ are always unstable with a linear growth rate proportional to $S^{-1 / 3}$ (here, $S$ is the Lundquist number), just as for the $m / n=1 / 1$ resistive kink mode. However, in our simulation model, the small amplitudes of $n=10$ modes at $t=0$ never catch up with the amplitude of $m / n=1 / 1$ mode, unless there is a triggering mechanism such as pressure steepening across the island separatrix. The phenomena is different from a mode- 
(a)

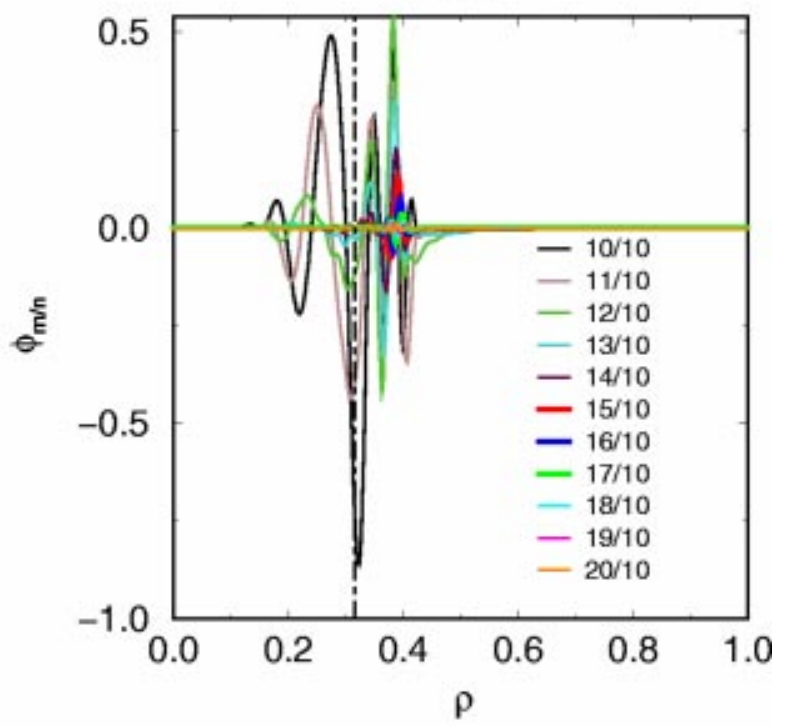

(b)

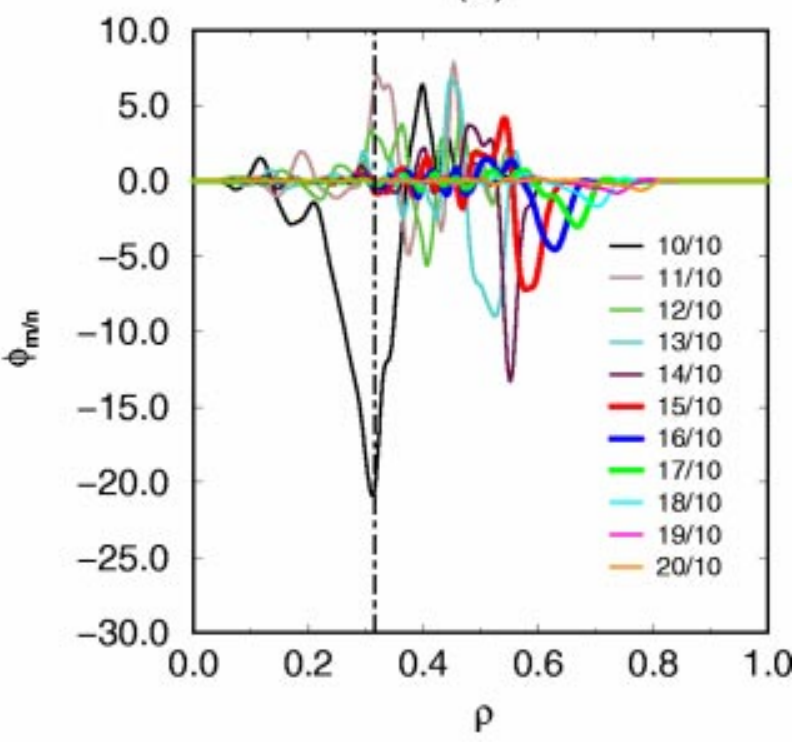

FIG. 4. Fourier spectrum of stream function $\phi$ (a) At $t=1.175 \times 10^{-2}$ and (b) at $t=1.230 \times 10^{-2}$. All modes shown have the same toroidal mode number $n=10$, but they have incommensurate helicities.

coupling energy cascade as one sees in magnetoturbulence. $^{23,24}$

Figure 5 shows the flow velocity field for the same $\beta=4 \%$ simulation. Interestingly, we can observe small scale eddy structures along the ballooning mode fingers streaming both inward and outward. Note that the significant eddy appears outside the $q=1$ radius, while similar structures exist inside the $q=1$ radius. These eddies convect plasma substantial distances across the $q=1$ surfaces.

In an actual plasma discharge, no modes can keep growing forever, unless, say, the entire plasma discharge is terminated. Any instability should reach a saturation stage. For example, in Fig. 2(f) the ballooning mode fingers are torn and we see a transition to another instability perhaps of a Kelvin-Helmholtz type. ${ }^{25}$ This signifies that numerous $m / n$ (a)

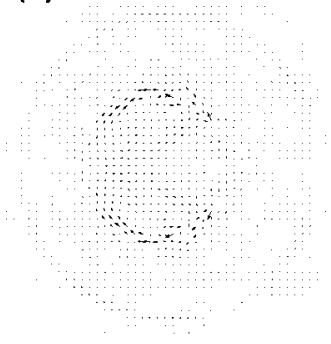

(c)

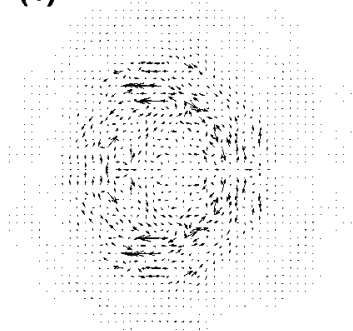

(e)

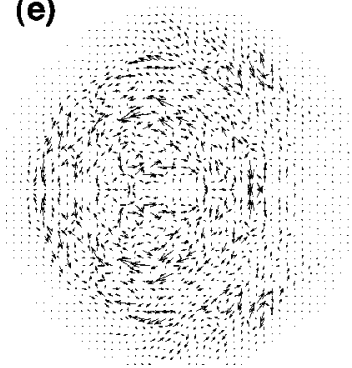

(b)

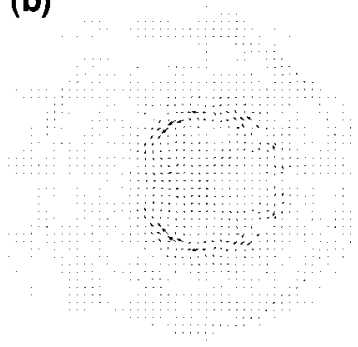

(d)

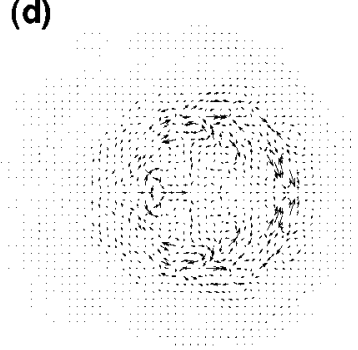

(f)
FIG. 5. The velocity field for the $\beta=4 \%$ simulation. The axisymmetry center line is located at the middle of the page. (a), (b) at $t=1.205 \times 10^{-2}$, (c), (d) at $t=1.230 \times 10^{-2}$, and (e), (f) at $t=1.265 \times 10^{-2}$. The lengths of the arrows are in the same unit for all the figures.

$\neq 1 / 1$ modes come into play and destroy the coherent structures. A key to understand "tearing" of the ballooning fingers is the finite dissipation. In a dissipative plasma, resistivity as well as viscosity plays a role in damping modes, especially in the short wavelength modes. As a reminder, the diffusion terms in Eqs. (5) and (6) are proportional to $\mathrm{m}^{2}$.

Figure 6 shows magnetic field line Poincaré plots during the ballooning mode evolution, at the time corresponding to those for the pressure contours of Fig. 2. As one can see, although the magnetic field lines are globally stochasticized, the field lines basically follow pressure surfaces. This is in contrast to the work by Kleva and Guzdar ${ }^{26}$ where the authors claim that the pressure and the magnetic field completely decouple and the pressure finger evolution does not affect the poloidal magnetic field strength. From the numerical measurement of the local magnetic field line pitch at $t=1.265 \times 10^{-2}$ we obtained $q_{0}<1$ near the shifted magnetic axis. The value of $q$-profiles for both the equilibrium (the solid line) and at the time $t=1.265 \times 10^{-2}$ (circles) are shown in Fig. 7. Given the location of the shifted magnetic axis $\left(X_{0}, Z_{0}\right)$ in a Cartesian coordinate system, a poloidal angle is calculated from 
(a)
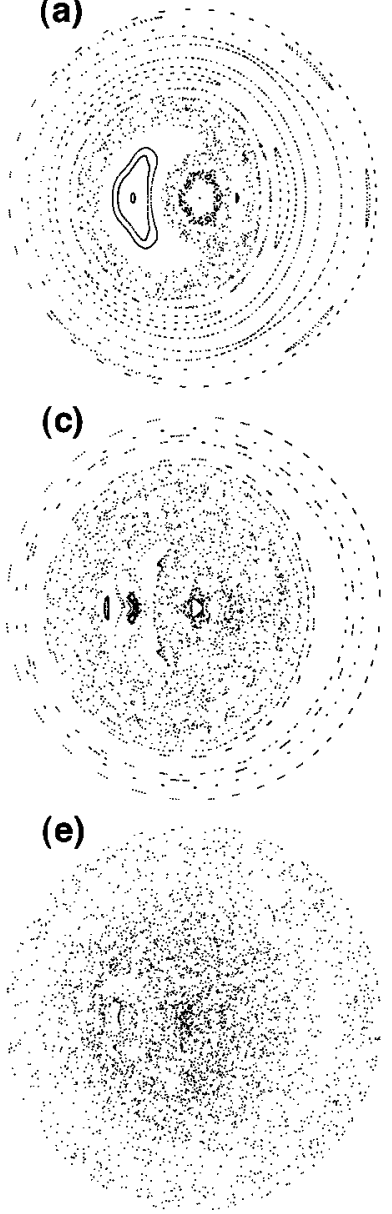
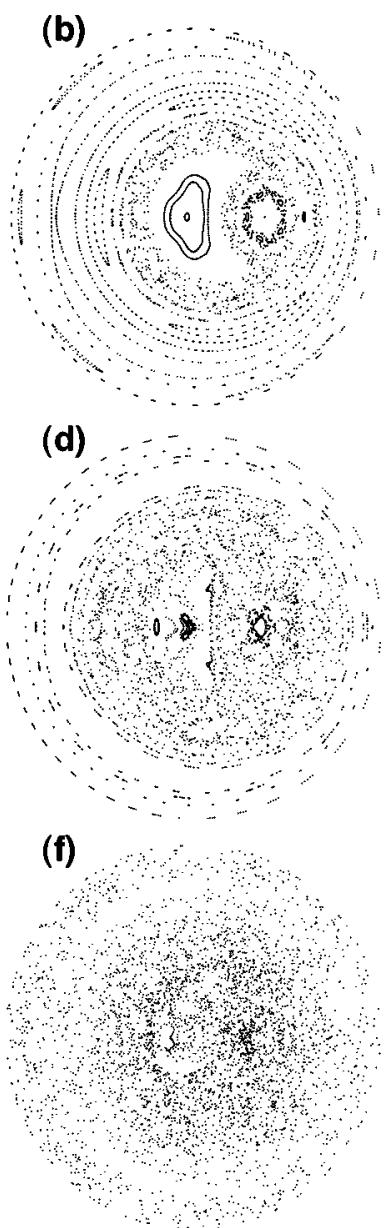

FIG. 6. Poincare plots of magnetic field line trajectories for the $\beta=4 \%$ simulation. The axisymmetry center line is located at the middle of the page. (a), (b) at $t=1.205 \times 10^{-2}$, (c), (d) at $t=1.230 \times 10^{-2}$, and (e), (f) at $t$ $=1.265 \times 10^{-2}$.

$$
\Theta=\arctan \frac{Z-Z_{0}(\zeta)}{X-X_{0}(\zeta)}
$$

Then, the safety factor is calculated by an averaged field line pitch

$$
\langle q\rangle=\lim _{\zeta \rightarrow \infty} \frac{\zeta}{\Theta(\zeta)-\Theta(0)} .
$$

The averaged $\langle q\rangle$-value in the perturbed state is no more than the ratio between the number of toroidal windings to poloidal windings. In Fig. 7, only five toroidal evolutions were taken for $\zeta$ in Eq. (7). If one follows field lines for a long period, the information of the initial position is lost due to magnetic stochasticity.

In Figs. 6(c)-6(f), global stochasticity can be seen in the annular region extending to $q \sim 2$ (most of the flux surface except for a few peripheral regions are destroyed). The $q$ $=1$ annular region is stochastic enough to produce rapid radial heat transport. (Note that parallel heat conduction is taken as zero in the work of this paper.) A simultaneous excitation of incommensurate high-n modes and the island overlapping allows rapid escape of the heat from the center to the $q \geqslant 1$ regions. This mode coupling feature of the bal-

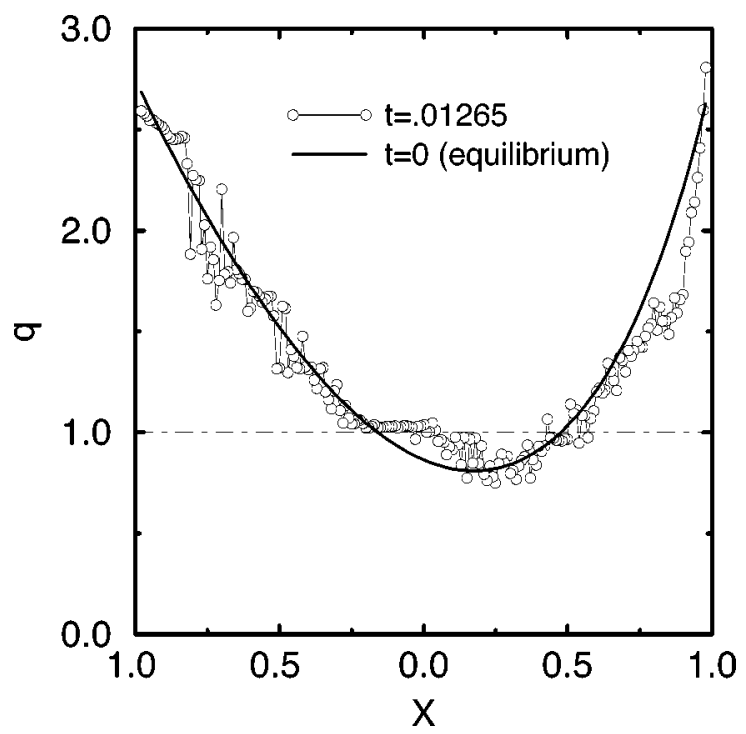

FIG. 7. The safety factor profile at the equilibrium (solid line) and at $t$ $=1.265 \times 10^{-2}$ (circles) which corresponds to Fig. 6(f).

looning modes and resultant global stochasticity can be a possible explanation for the rapid escape of the heat observed in TFTR experiment. ${ }^{10}$ As a result, after the stage of Figs. 2(e) and 2(f), the effect of finite parallel heat transport along the stochastic magnetic field lines becomes dominant for the pressure evolution. The inclusion of parallel heat transport effects into the MHD model is numerically too demanding and we do not present these simulation results in this paper.

With a decrease in $\beta(4 \% \rightarrow 2 \%$, see Fig. 8), the ballooning structures can be still seen, but they are rather modest ( $\beta_{p}=0.49$ for $\beta=2 \%$ ). The onset of the ballooning instability is delayed for this lower $\beta$ case; finger-like structures appear at a much later phase of the $m=1$ island evolution. It takes a longer time for the pressure gradient to build up to the ballooning threshold. Another observation is the degree of radial extension of the fingers. In the $\beta=2 \%$ case, the finger extends only up to $q \sim 1.3$ surface. As a result, the extension of stochastic annular region is modest compared to higher $\beta$ cases. Figure 9 shows magnetic field line Poincaré plots during the ballooning mode evolution, at the time corresponding to those for the pressure contours of Fig. 8 .

In the lower $\beta\left(\beta_{p}<0.25\right)$ limit, the magnetic field lines seem to recover the Kadomtsev-type full magnetic reconnection. As a reminder, the TEXTOR ${ }^{11}$ experiments are conducted in $\beta_{p} \leqslant 0.3$ and the work by Nagayama et al. in TFTR $^{6}$ experiments reveals $\beta_{p} \sim 0.95$ for the hot ion mode, $\beta_{p} \sim 0.4$ for the ion cyclotron range of frequencies (ICRF) plasmas, and $\beta_{p} \sim 0.11$ for the Ohmic plasmas. Although magnetic stochasticity induced by the ballooning modes may play a role, the mechanism for the incomplete magnetic reconnection process in the low- $\beta$ plasmas remains to be explored.

\section{TOROIDAL ASYMMETRY OF THE $\boldsymbol{m}=1$ MAGNETIC ISLAND}

For the simulation in this section, we have taken the equilibrium $q$ profile and the pressure profile (the equilib- 
(a)

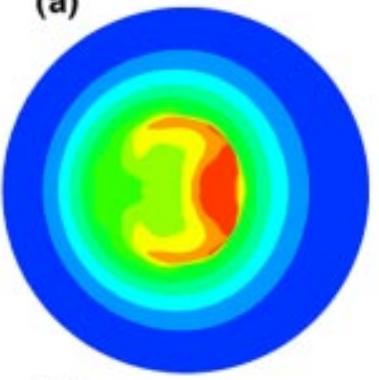

(c)

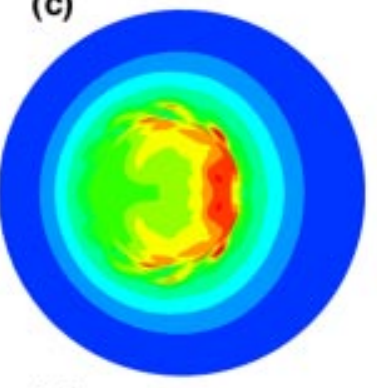

(e)

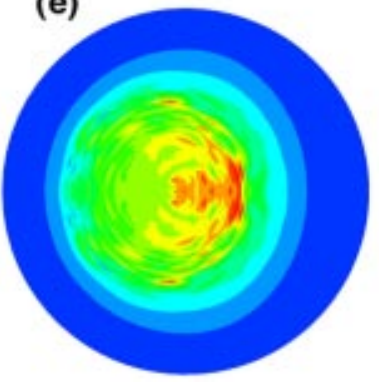

(b)

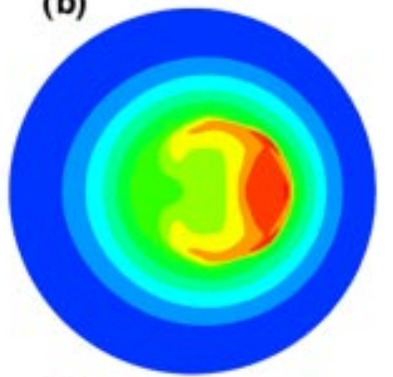

(d)

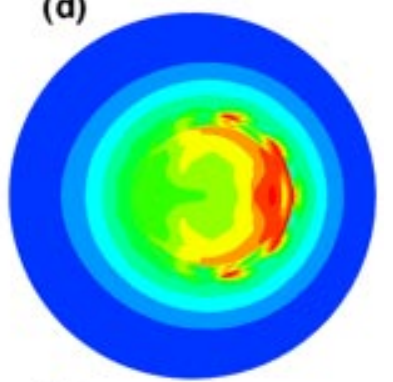

(f)

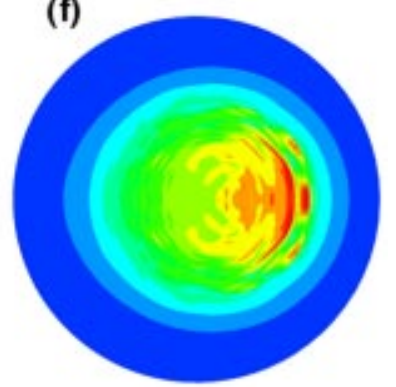

FIG. 8. Pressure contours for the $\beta=2 \%$ simulation. The axisymmetry center line is located at the middle of the page. (a), (b) at $t=1.162 \times 10^{-2}$, (c), (d) at $t=1.187 \times 10^{-2}$, and (e), (f) at $t=1.225 \times 10^{-2}$.

rium of $\beta=4 \%$ ) as in the previous section. However, we do not include pressure/curvature effects in the mode evolution and we set $\beta=0$ in Eq. (5). Thus, the plasma responds to a Shafranov shift (which is $17 \%$ of the minor radius for the equilibrium employed here) and the equilibrium geometry metric elements, but the dynamical ballooning mode evolution is absent. The aim of this rather artificial calculation is to extract the magnetic field line structure near the $m=1$ island separatrix or the current sheet, until the moment of the ballooning modes onset. As shown in Figs. 6 and 9, once the short wavelength ballooning modes are triggered, the magnetic field lines basically follow the pressure surface which exhibits the finger-like structure. As suggested by Hegna and Callen $^{27}$ the local magnetic shear can be an important factor for the triggering of ballooning modes. The ballooning modes can become unstable when the destabilizing pressuredrive overcomes the field line bending stabilizing contribution on the perturbed magnetic surface. ${ }^{27}$ Inclusion of a dynamical curvature term generates magnetic stochasticity and makes observation of the magnetic structures very difficult. Here, we have also employed an extremely large viscosity value $\left(\nu_{\perp}=5.0\right)$ so as to kill the strong flow.

Figure 10 shows Poincaré plots of magnetic field line trajectories when the hot core is facing the good (left) and

(a)

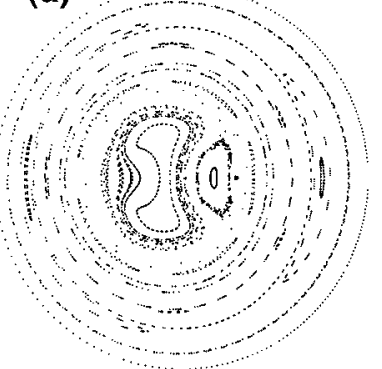

(c)

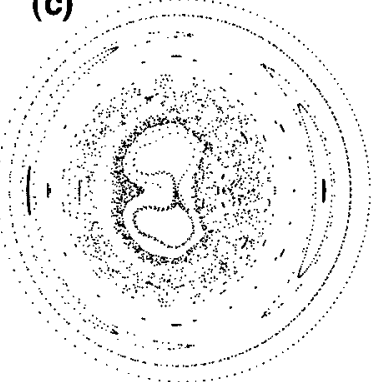

(e)

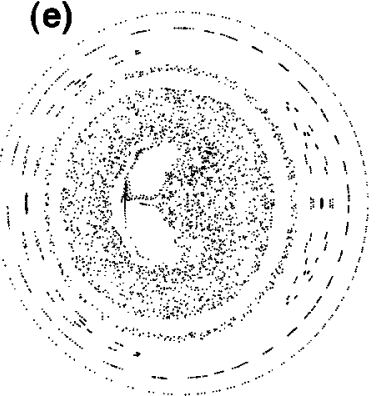

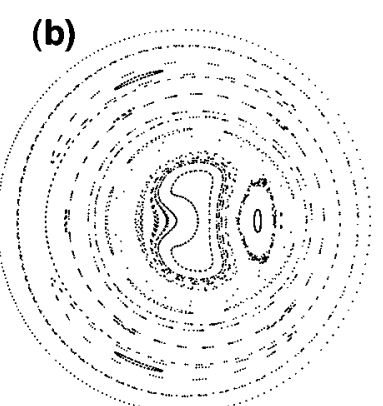

(d)
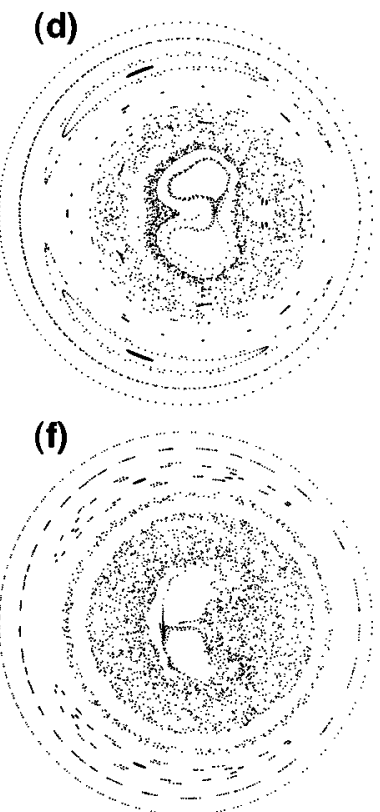

FIG. 9. Poincaré plots of magnetic field line trajectories for the $\beta=2 \%$ simulation. The axisymmetry center line is located at the middle of the page. (a), (b) at $t=1.162 \times 10^{-2}$, (c), (d) at $t=1.187 \times 10^{-2}$, and (e), (f) at $t=1.225 \times 10^{-2}$

the bad (right) curvature sides of the plasma. As before, the center line is located at the middle of the figure, and the toroidal magnetic field falls off as $\sim 1 / R$ from the center toward edges of the figure. By comparing Poincaré mappings of the field line trajectories on the good and bad curvature sides, toroidal asymmetry in the magnetic field structure can be seen: the internal column is close to the wall in Fig. 10(b) when the hot core region faces the bad curvature side. One can observe the three-dimensional structure of the current sheet $^{28}$ of the $m=1$ magnetic island (or the Y-ribbons ${ }^{19}$ ); the compression of the flux surfaces is much more pronounced at a toroidal angle where the current sheet is located on the bad curvature side of the torus. The central core has an oval shape rather than circular. The oval shape is upright when facing the bad curvature side, but horizontal when facing the good curvature side. As shown in Figs. 10(e) and 10(f), Kadomtsev-type full magnetic reconnection ${ }^{4}$ takes place in the absence of dynamical ballooning modes.

From this magnetic structure, one can expect that the resultant ballooning-type instabilities across the $q=1$ surface will be toroidally localized by their alignment with the magnetic island structure. This mechanism is not the same as the localization of the ballooning modes which is demonstrated in the previous section. This mechanism arises from the geo- 

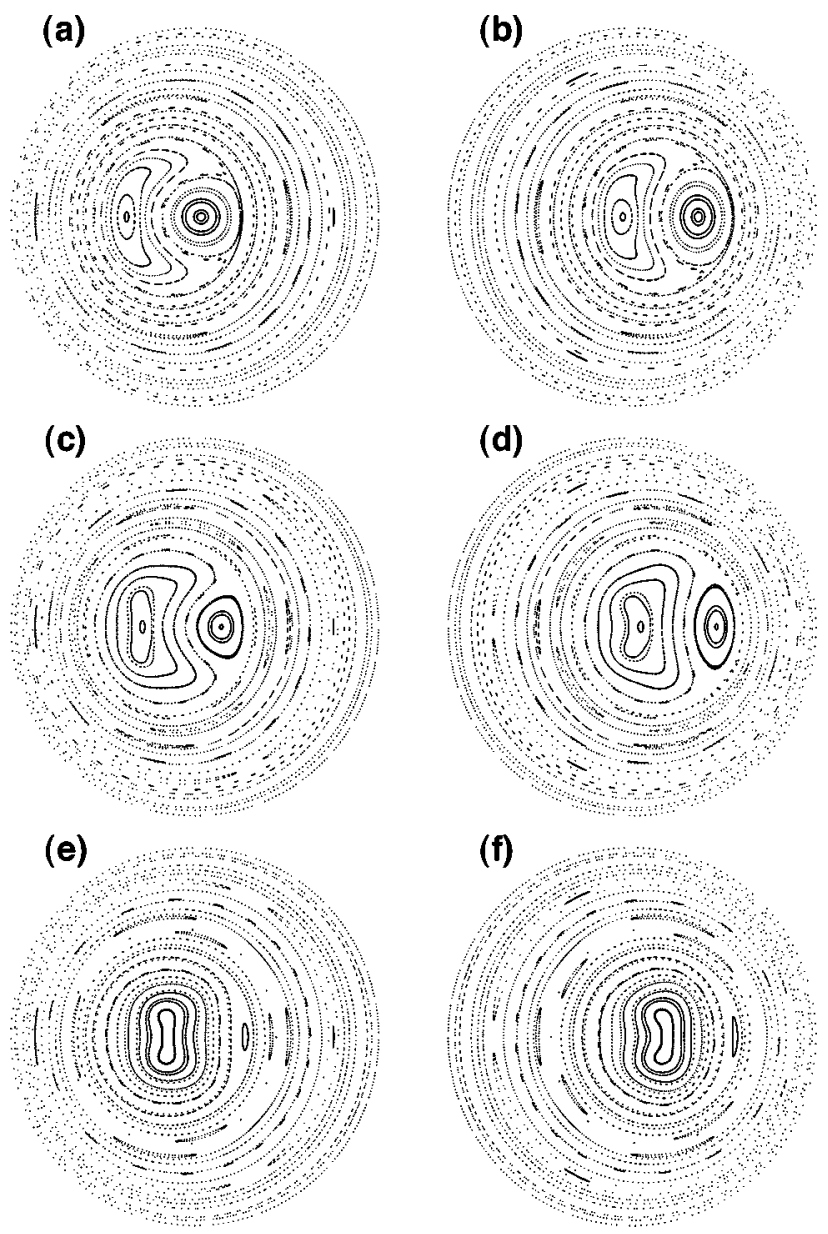

FIG. 10. Poincare plots of magnetic field line trajectories for the $\beta=4 \%$ simulation. Kadomtsev type full reconnection takes place in the absence of dynamical ballooning modes. The axisymmetry center line is located at the middle of the page. (a), (b) at $t=2.52 \times 10^{-2}$, (c), (d) at $t=2.56 \times 10^{-2}$, and (e), (f) at $t=2.60 \times 10^{-2}$.

metrical effects on the $m / n=1 / 1$ magnetic island evolution due to the $1 / R$ dependence of the toroidal magnetic field.

\section{SUMMARY AND DISCUSSION}

In this paper, the tokamak sawtooth crash phase has been studied numerically employing a toroidal magnetohydrodynamic initial value simulation. Motivated by experimental results from TFTR showing dramatic temperature evolution during the crash, emphasis has been put on the role of pressure driven high- $n$ ballooning modes.

With a wide selection of Fourier modes, it has been shown that the $m / n=1 / 1$ magnetic reconnection process induces nonlinearly unstable high-n ballooning modes; the $m / n=1 / 1$ magnetic island modifies the pressure profiles and generates unstable states for ballooning modes. The ballooning spectrum in the simulation compares favorably with that expected from conventional MHD theory; ${ }^{20}$ all the modes with different $m$ numbers but with the same $n$ mode numbers are correlated. In the crash phase, it is observed that small scale vortices are generated. The results were significant in suggesting the breaking of the symmetric $m=1$ flows, and hence the possibility of temperature profile flattening without complete magnetic reconnection in tokamak plasma discharges.

As an artificial probe for the onset mechanism of high- $n$ ballooning modes, the $m / n=1 / 1$ island evolution for a large equilibrium $\beta$ value (and thus a large Shafranov shift) has been simulated but without the dynamical pressure-drive. By comparing the magnetic field line trajectories on the good and bad curvature sides, we have observed a threedimensional, non-axisymmetric structure of the $m=1 \mathrm{mag}$ netic island. It has been shown that the current sheet, ${ }^{28}$ or the Y-ribbon, ${ }^{19}$ is stretched (vertically elongated) when it is facing the bad curvature side. The effect is considered to induce ballooning modes on the bad curvature side.

Compared to previous work by Park et al. ${ }^{29}$ where the helically twisted equilibrium produced by the kink mode was taken as an initial configuration, this is the first MHDsimulation to reveal the activity of the secondary high- $n$ ballooning modes, starting from a self-consistent concentric equilibrium that evolved into an $m / n=1 / 1$ magnetic island structure. The simulation by Park et al. ${ }^{29}$ was conducted to account for the TFTR $\beta$ limit disruptions ${ }^{30}$ rather than sawtooth crashes. In the $\beta$ limit disruptions ${ }^{30}$ it is observed that the ideal kink modes (instead of resistive kink modes) give rise to precursor signals and the 1/1 kink strongly couples to higher $m / n$ components. (It is worth pointing out that the localization of a pressure hot bulge to the bad curvature side during the $m / n=1 / 1$ island evolutions has been demonstrated by Park et al. ${ }^{31}$ and Aydemir. ${ }^{32}$ ) To emphasize again, in our studies, the high- $n$ ballooning activity does not come from the mode couplings. The ballooning modes are suddenly triggered due to the emergence of the magnetic island or the "topological change in magnetic field lines" and the local pressure steepening.

With regard to magnetic structures, it has been shown that the ballooning mode can break the helical symmetry and thereby induce magnetic stochasticity. In the high- $\beta$ simulations, significant stochasticity has been induced by the strong mode couplings between modes of incommensurate helicity, which can induce radial thermal transport along the field lines. This supports TFTR experimental results which suggest rapid heat escape from the center to the outside $q=1$ region. ${ }^{10}$

The relation of our simulation results to the experimentally observed partial reconnection in $\mathrm{TFTR}^{6}$ is discussed here. As indicated experimentally ${ }^{10}$ the flattening of the temperature profile takes place before the full reconnection of the magnetic field lines. We suggest that, in relatively high- $\beta$ plasmas, the ballooning modes are playing a role in the flattening of the temperature crash in the nonlinear stage of sawtooth events. The dominant mode is converted from the $m / n=1 / 1$ mode to $n=10$ higher harmonics (via onset of high- $n$ ballooning modes). Furthermore, we conjecture that the resultant small scale vortices may be playing an important role in the mixing and at the same time damping of strong $m=1$ flow. At the temperature crash phase, we have shown that the local magnetic field line pitch at the magnetic axis stays as $q_{0}<1$. Though the parallel heat transport along the stochastic magnetic field lines becomes dominant for the 
pressure evolution after the temperature crash, we did not simulate the effect in this paper. The heat conduction effects parallel to the magnetic field lines remain to be explored, since, in the lower $\beta$ limit $\left(\beta_{p} \leqslant 0.25\right)$, our simulation results seem to recover the Kadomtsev type full magnetic reconnection, while the experimental results from TEXTOR ${ }^{11}$ and TFTR $^{10}$ typically reveal the partial magnetic reconnection.

The experimental sawteeth analyses by Nagayama et al. ${ }^{6}$ exhibit the localization of the ballooning modes on the bad curvature side in high- $\beta$ TFTR discharges. Our simulation results of ballooning mode in high $\beta\left(\beta>2 \%\right.$ and $\beta_{p}$ $>0.49$ ) have common features with their experimental findings. On the other hand, as shown in Figs. 6 and 9, the magnetic topology at the both sides of the torus seems to be the same, and did not reveal the evidence of threedimensional reconnection model proposed by Nagayama et $a l .{ }^{6}$ We suspect this discrepancy can be due to the reduced MHD formulation we employed and the lack of stabilizing parallel heat transport effects along stochastic magnetic field lines (the possibility of the three-dimensional reconnection process still remains).

Collisionless magnetic reconnection model has been developed ${ }^{33,34}$ to explain fast reconnection including nonideal kinetic effects in the thin current layer. Our numerical simulation results are antithetical to those models in that high- $n$ ballooning modes across the current layer can destroy effects such as electron inertia. It is difficult to imagine that the electron current sheet is not being influenced by the pressure-drive effects which cause very strong radial flows.

As a summary, this paper has demonstrated an important nature of the high- $n, m / n \neq 1 / 1$ mode activities during the tokamak sawtooth crash in relatively high- $\beta$ plasmas. We further investigated the strong interaction between the $m$ $=1$ flow and the high- $n$ mode flows. Further progress in the magnetic reconnection model will be reported in the future.

\section{ACKNOWLEDGMENTS}

One of the authors (YN) is grateful to Dr. B. Coppi, Dr. Y. Nagayama, Dr. W. Park, and Dr. M. Yamada for valuable suggestions. Y.N. would like to acknowledge an enormous credit from Dr. B. A. Carreras, Dr. L. A. Charlton, Dr. H. R. Hicks, Dr. J. A. Holmes, Dr. J-N. G. Leboeuf, Dr. D. K. Lee, Dr. V. E. Lynch, Dr. D. A. Spong, and Dr. B. V. Waddell for the development of FAR.

This research was supported by United States Department of Energy Grant No. DE-FG02-86ER53218.

${ }^{1}$ S. von Goeler, W. Stodiek, and N. Sauthoff, Phys. Rev. Lett. 33, 1201 (1974).

${ }^{2}$ H. Soltwisch, W. Stodiek, J. Manickam, and J. Schlüter, in Plasma Physics and Controlled Nuclear Fusion Research, 1986, Proceedings of the 11th International Conference, Kyoto (International Atomic Energy Agency, Vienna, 1987), Vol. 1, p. 263; H. Soltwisch, Rev. Sci. Instrum. 59, 1599 (1988)
${ }^{3}$ F. M. Levinton, L. Zakharov, S. H. Batha, J. Manickam, and M. C. Zarnstorff, Phys. Rev. Lett. 72, 2895 (1994).

${ }^{4}$ B. B. Kadomtsev, 1, 389 (1975); B. B. Kadomtsev, in Plasma Physics and Controlled Nuclear Fusion Research, 1976, Proceedings of the 6th International Conference, Berchtesgaden (International Atomic Energy Agency, Vienna, 1977), Vol. 1, p. 555.

${ }^{5}$ E. N. Parker, Astrophys. J. 121, 491 (1955); P. A. Sweet, Nuovo Cimento Suppl. 8, 188 (1958).

${ }^{6}$ Y. Nagayama, M. Yamada, W. Park, E. D. Fredrickson, A. C. Janos, K. M. McGuire, and G. Taylor, Phys. Plasmas 3, 1647 (1996).

${ }^{7}$ Y. Nagayama, K. M. McGuire, M. Bitter, A. Cavallo, E. D. Fredrickson, K. W. Hill, W. Park, G. Taylor, and M. Yamada, Phys. Rev. Lett. 67, 3527 (1991).

${ }^{8}$ J. D. Callen, B. A. Carreras, and R. D. Stambaugh, Phys. Today 45, 34 (1992).

${ }^{9}$ E. D. Fredrickson, K. McGuire, A. Cavallo, R. Budny, A. Janos, D. Monticello, Y. Nagayama, W. Park, G. Taylor, and M. C. Zarnstorff, Phys. Rev. Lett. 65, 2869 (1990).

${ }^{10}$ M. Yamada, F. Levinton, N. Pomphrey, R. Budny, J. Manickam, and Y. Nagayama, Phys. Plasmas 1, 3269 (1994).

${ }^{11}$ H. Soltwisch and H. R. Koslowski, Plasma Phys. Controlled Fusion 37, 667 (1995).

${ }^{12}$ Y. Nishimura, Ph.D. dissertation, University of Wisconsin-Madison, 1998.

${ }^{13}$ H. R. Strauss, Phys. Fluids 20, 1354 (1977).

${ }^{14}$ V. E. Lynch, B. A. Carreras, H. R. Hicks, J. A. Holmes, and L. Garcia, Comput. Phys. Commun. 24, 465 (1981).

${ }^{15}$ L. A. Charlton, J. A. Holmes, H. R. Hicks, V. E. Lynch, and B. A. Carreras, J. Comput. Phys. 63, 107 (1986).

${ }^{16}$ M. N. Bussac, R. Pellat, D. Edery, and J. L. Soule, Phys. Rev. Lett. 35, 1638 (1975).

${ }^{17}$ M. Abramowitz and I. A. Stegun, Handbook of Mathematical Functions (Dover, New York, 1970), p. 896. A better fourth order Runge-KuttaGill method which includes corrections to the truncation error can be found in I. Kawakami, Suuchi-keisan (Iwanami, Tokyo, 1989), p. 159 (in Japanese).

${ }^{18}$ H. P. Furth, P. H. Rutherford, and H. Selberg, Phys. Fluids 16, 1054 (1973).

${ }^{19}$ F. L. Waelbroeck, Phys. Fluids B 1, 2372 (1989).

${ }^{20}$ J. W. Connor, R. J. Hastie, and J. B. Taylor, Phys. Rev. Lett. 40, 396 (1978).

${ }^{21}$ M. Abramowitz and I. A. Stegun, Handbook of Mathematical Functions (Dover, New York, 1970), p. 498.

${ }^{22}$ R. B. White, Theory of Tokamak Plasmas (North-Holland, Amsterdam, 1989), p. 192.

${ }^{23}$ D. Biskamp, Nonlinear Magnetohydrodynamics (Cambridge, London, 1993), p. 183

${ }^{24}$ K. Hallatschek, A. Gude, D. Biskamp, S. Günter, and ASDEX Upgrade Team, Phys. Rev. Lett. 80, 293 (1998).

${ }^{25} \mathrm{~S}$. Chandrasekhar, Hydrodynamic and Hydromagnetic Stability (Clarendon, Oxford, 1961), p. 507.

${ }^{26}$ R. G. Kleva and P. N. Guzdar, Phys. Rev. Lett. 80, 3081 (1998).

${ }^{27}$ C. C. Hegna and J. D. Callen, Phys. Fluids B 4, 3031 (1992).

${ }^{28}$ M. N. Rosenbluth, R. Y. Dagazian, and P. H. Rutherford, Phys. Fluids 16, 1894 (1973).

${ }^{29}$ W. Park, E. D. Fredrickson, A. Janos, J. Manickam, and W. M. Tang, Phys. Rev. Lett. 75, 1763 (1995).

${ }^{30}$ E. D. Fredrickson, K. McGuire, Z. Chang, A. Janos, M. Bell, R. V. Budny, C. E. Bush, J. Manickam, H. Mynick, R. Nazikian, and G. Taylor, Phys. Plasmas 2, 4216 (1995).

${ }^{31}$ W. Park, D. A. Monticello, E. D. Fredrickson, and K. McGuire, Phys. Fluids B 3, 507 (1991).

${ }^{32}$ A. Aydemir, Phys. Fluids B 2, 2135 (1990).

${ }^{33}$ J. A. Wesson, Nucl. Fusion 30, 2545 (1990).

${ }^{34}$ D. Biskamp and J. F. Drake, Phys. Rev. Lett. 73, 971 (1994). 